\title{
Quantum imaging with incoherently scattered light from a free-electron laser
}

\author{
Raimund Schneider ${ }^{1,2}$, Thomas Mehringer ${ }^{1,2}$, Giuseppe Mercurio ${ }^{3,4}$, Lukas Wenthaus ${ }^{3,4}$, \\ Anton Classen ${ }^{1,2}$, Günter Brenner ${ }^{5}$, Oleg Gorobtsov ${ }^{5}$, Adrian Benz ${ }^{3}$, Daniel Bhatti, ${ }^{1,2}$, Lars Bocklage ${ }^{5,6}$, \\ Birgit Fischer $^{7}$, Sergey Lazarev ${ }^{5,8}$, Yuri Obukhov ${ }^{9}$, Kai Schlage ${ }^{5}$, Petr Skopintsev ${ }^{5}$, Jochen Wagner ${ }^{10}$, \\ Felix Waldmann ${ }^{1}$, Svenja Willing ${ }^{5}$, Ivan Zaluzhnyy ${ }^{5,11}$, Wilfried Wurth ${ }^{3,4,5}$, Ivan A. Vartanyants ${ }^{5,11}$, \\ Ralf Röhlsberger ${ }^{5,6}$ and Joachim von Zanthier ${ }^{1,2 \star}$
}

\begin{abstract}
The advent of accelerator-driven free-electron lasers (FEL) has opened new avenues for high-resolution structure determination via diffraction methods that go far beyond conventional $\mathrm{X}$-ray crystallography methods ${ }^{1-10}$. These techniques rely on coherent scattering processes that require the maintenance of first-order coherence of the radiation field throughout the imaging procedure. Here we show that higher-order degrees of coherence, displayed in the intensity correlations of incoherently scattered X-rays from an FEL, can be used to image two-dimensional objects with a spatial resolution close to or even below the Abbe limit. This constitutes a new approach towards structure determination based on incoherent processes ${ }^{11,12}$, including fluorescence emission or wavefront distortions, generally considered detrimental for imaging applications. Our method is an extension of the landmark intensity correlation measurements of Hanbury Brown and Twiss $^{13}$ to higher than second order, paving the way towards determination of structure and dynamics of matter in regimes where coherent imaging methods have intrinsic limitations ${ }^{14}$.

The discovery by Hanbury Brown and Twiss of photon bunching of thermal light ${ }^{15}$ and its application in astronomy to determine the angular diameter of stars by measuring spatial photon correlations ${ }^{13}$ was a hallmark experiment for the development of modern quantum optics $^{16}$. The subsequent quantum mechanical description of photon correlations by Glauber paved the way for a generalized concept of optical coherence ${ }^{17}$ that is founded on the analysis of correlation functions of order $m$ rather than the first-order coherence. For example, the spatial second-order photon correlation function $g^{(2)}\left(\mathbf{r}_{1}, \mathbf{r}_{2}\right)$ expresses the probability to detect a photon at position $\mathbf{r}_{1}$ given that a photon is recorded at position $\mathbf{r}_{2}$. In the case of two incoherent sources, $g^{(2)}\left(\mathbf{r}_{1}, \mathbf{r}_{2}\right)$ displays a cosine modulation which oscillates at a spatial frequency depending on the source separation $^{18,19}$. In this way interference fringes show up even in the complete absence of first-order coherence, allowing the extraction of structural information from incoherently emitting objects. This has
\end{abstract}

been applied in Earth-bound stellar interferometry to measure the angular diameter of stars with 100 -fold increased resolution ${ }^{13}$ or to reveal the spatial and statistical properties of pulsed FEL sources ${ }^{20,21}$.

Extending this concept to arbitrary arrangements of incoherently scattering emitters enables one to use intensity correlations for imaging applications. This has been demonstrated recently for one-dimensional arrays of emitters in the visible range of the spectrum $^{22-24}$, where a spatial resolution even below the canonical Abbe limit has been achieved. Here we go still further and employ the method to image arbitrary two-dimensional incoherently scattering objects radiating in the vacuum ultraviolet. The extension from one dimension $^{24}$ to two dimensions is non-trivial and even unexpected in view of the tremendously enlarged parameter space for the possible phase combinations determining the higher-order correlation functions. It constitutes a major breakthrough of the scheme as it allows application of the method for real imaging applicationsfor example, imaging of arbitrary two-dimensional (2D) objects on a substrate. Furthermore, being able to measure 2D intensity correlations of order $m>2$ in the X-ray domain demonstrates that ideas of quantum imaging, developed at visible wavelengths, can be transferred to shorter wavelengths, despite substantially reduced signal-to-noise ratios (in our case by four orders of magnitude) due to significantly decreased levels of scattered light.

The experiment has been performed at the PG2 beamline of the FLASH FEL facility at DESY, Hamburg ${ }^{25}$. The scheme of the set-up is shown in Fig. 1a. The FEL runs in a $10 \mathrm{~Hz}$ pulsed mode ( $\tau_{\text {pulse }} \approx 60 \mathrm{fs}$ ) at a centre wavelength of $\lambda=13.2 \mathrm{~nm}$ (for experimental details see ref. 21). After passing a monochromator to limit the bandwidth to $0.1 \%$ ( $\pm 0.013 \mathrm{~nm}$ ), the FEL beam impinges on a slowly moving diffusor converting the spatially highly coherent light of the FEL to quasi-monochromatic pseudo-thermal light with a far-field speckle pattern that fluctuates randomly from shot to shot but is stationary for each pulse $\mathrm{e}^{26}$. This ensures that any sets of scatterers (or holes) illuminated by the light field from the diffusor act as ensembles of incoherent sources emitting

\footnotetext{
${ }^{1}$ Institut für Optik, Information und Photonik, Universität Erlangen-Nürnberg, Staudtstraße 1, 91058 Erlangen, Germany. ${ }^{2}$ Erlangen Graduate School in Advanced Optical Technologies (SAOT), Universität Erlangen-Nürnberg, Paul-Gordan-Straße 6, 91052 Erlangen, Germany. ${ }^{3}$ Department Physik, Universität Hamburg, Luruper Chaussee 149, 22761 Hamburg, Germany. ${ }^{4}$ Center for Free-Electron Laser Science, Luruper Chaussee 149, 22761 Hamburg, Germany. ${ }^{5}$ Deutsches Elektronen-Synchrotron DESY, Notkestraße 85, 22607 Hamburg, Germany. ${ }^{6}$ The Hamburg Centre for Ultrafast Imaging, Luruper Chaussee 149, 22761 Hamburg, Germany. ${ }^{7}$ Institut für Physikalische Chemie, Universität Hamburg, Grindelallee 117, 20146 Hamburg, Germany. ${ }^{8}$ National Research Tomsk Polytechnic University (TPU), Prospekt Lenina 30, 634050 Tomsk, Russia. ${ }^{9}$ Nuclear Safety Institute, Russian Academy of Sciences, Bolshaya Tulskaya ul. 52, 115191 Moscow, Russia. ${ }^{10}$ Institut für Nanostruktur- und Festkörperphysik, Universität Hamburg, Luruper Chaussee 149, 22761 Hamburg, Germany. ${ }^{11}$ National Research Nuclear University MEPhl (Moscow Engineering Physics Institute), Kashirskoe shosse 31, Moscow 115409, Russia. *e-mail: joachim.vonzanthier@fau.de
} 
a

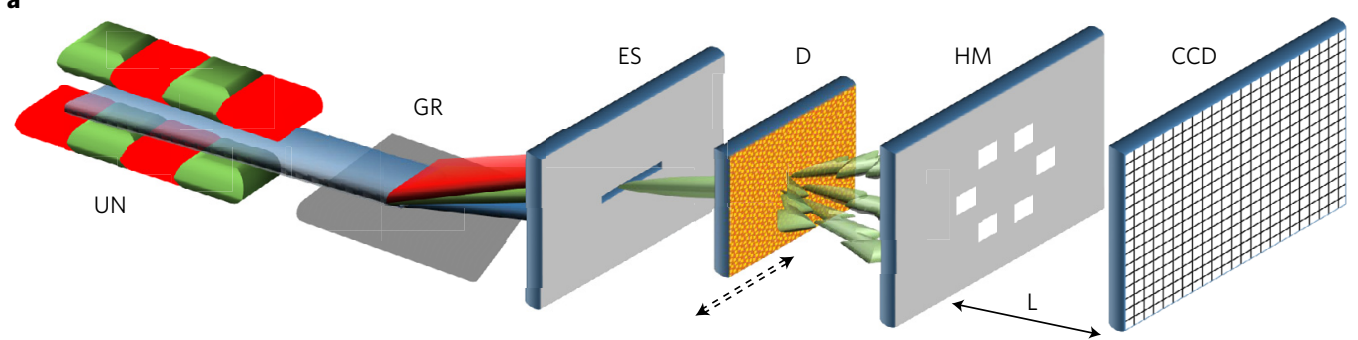

b

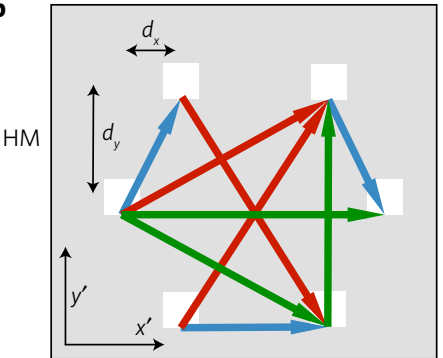

CCD

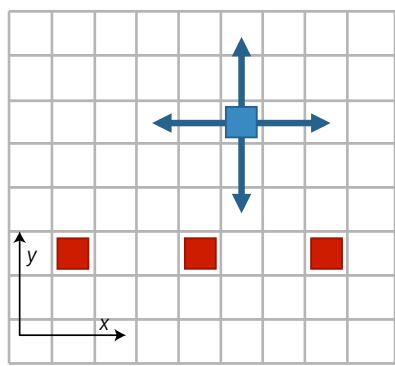

Figure 1 | Scheme of the experiment. $\mathbf{a}$, The FEL beam created in the undulator (UN) passes a monochromator consisting of a grating (GR) and an exit slit (ES) such that quasi-monochromatic coherent radiation impinges on the moving diffusor (D). The pseudo-thermal light scattered by the diffusor is used to illuminate a benzene-like hole mask (HM) generating six quasi-monochromatic independently radiating incoherent light sources. The resulting light fields are measured at a distance $L$ behind HM by a CCD. $\mathbf{b}$, The nine different source separation vectors $\mathbf{h}_{i}, i=1, \ldots 9$, of $\mathrm{HM}$ are shown in different colours for better readability. $\mathbf{c}$, To determine $g^{(m)}\left(\mathbf{r}_{1} ; M P_{x}\right)$, for each CCD image, $m-1$ pixels of the CCD camera are selected at the magic positions along the $x$-axis (red) and correlated with all other pixels (blue) of the CCD; thereafter an average over all images is taken.

independently from each other (see Supplementary Information). In our experiment, a two-dimensional object mask is placed behind the diffusor, consisting of six square-cut holes in a hexagonal arrangement to mimic the carbon atoms in a benzene molecule emitting incoherent fluorescence radiation. The structure of this object is characterized by a set of nine spatial frequency vectors $\zeta=\left\{\mathbf{f}_{i}=\left(f_{i, x}, f_{i, y}\right)=\left(h_{i, x} /(\lambda L), h_{i, y} /(\lambda L)\right), i=1, \ldots, 9\right\}$, determined by the nine different source separation vectors $\mathbf{h}_{i}=\left(h_{i, x}, h_{i, y}\right)$, $i=1, \ldots 9$, connecting the artificial atoms (that is, the holes in the mask), as shown in Fig. 1b. The light passing through the object mask is measured by a charge-coupled device (CCD) camera in the far field at a distance $L=275 \mathrm{~mm}$ behind the source plane, where each pixel of the CCD serves as an independent detector (see Fig. 1b).

The resulting intensity patterns are recorded in the far field for each pulse individually (see Fig. 2a). By correlating the intensities $I\left(\mathbf{r}_{i}\right)$ at different positions $\mathbf{r}_{i}, i=1, \ldots, m$, for each pulse and averaging the result over many pulses we obtain the $m$ th-order intensity correlation function

$$
g^{(m)}\left(\mathbf{r}_{1}, \ldots, \mathbf{r}_{m}\right)=\frac{\left\langle I\left(\mathbf{r}_{1}\right) \cdots I\left(\mathbf{r}_{m}\right)\right\rangle}{\left\langle I\left(\mathbf{r}_{1}\right)\right\rangle \cdots\left\langle I\left(\mathbf{r}_{m}\right)\right\rangle}
$$

Our imaging algorithm aims to extract the set of spatial frequency vectors $\zeta$ of the unknown source distribution-assumed to be placed on a $2 \mathrm{D}$ lattice-from the higher-order intensity correlations $g^{(\mathrm{m})}$; the source geometry in real space is then reconstructed from $\zeta$ (see Supplementary Information). In the intensity correlations of second order the entire set of spatial frequency vectors appears, hence the number of parameters to be fitted and extracted from $g^{(2)}$ is particularly large, especially in the case of a data set with low signal-to-noise ratio. In contrast, measuring intensity correlations of higher than second order allows one to split the information, as only a subset of spatial frequencies appears within a given correlation order $m$ (see below). This leads to a significantly reduced number of fit parameters to be determined from each correlation function $g^{(m)}$, allowing one to reveal $\zeta$ with substantially increased accuracy ${ }^{24}$. In certain cases even a numerical aperture smaller than required by the canonical Abbe limit allows one to determine $\zeta$, leading to a spatial resolution below the classical diffraction limit. This will be the case if the frequency vector $\left(\begin{array}{l}0 \\ 1\end{array}\right)$ or $\left(\begin{array}{l}1 \\ 0\end{array}\right)$, corresponding to the smaller of the two grid constants $d_{x}$ and $d_{y}$, is part of the complete set of spatial frequencies $\zeta$ (ref. 24) (see Supplementary Information). For the particular source arrangement considered in our experiment (that is, the hexagonal structure in the form of a benzene-like molecule) this is not the case. However, we emphasize that ultimately, at very high photon energies, the benefit of our scheme lies less in obtaining a resolution below the Abbe limit, but in gaining information about the structure and dynamics of matter when coherent imaging methods fail or have intrinsic limitations ${ }^{14}$.

The $m$ th-order spatial intensity correlation functions $g^{(m)}\left(\mathbf{r}_{1}, \ldots, \mathbf{r}_{m}\right)$ are computed by correlating the intensities measured by $m$ pixel detectors at positions $\mathbf{r}_{i}, i=1, \ldots, m$, for each CCD image individually and then averaging over all CCD images (see Supplementary Information). In general $g^{(m)}\left(\mathbf{r}_{1}, \ldots, \mathbf{r}_{m}\right)$ takes a complicated form, depending on the detector positions $\mathbf{r}_{1}, \ldots, \mathbf{r}_{m}$ and the source geometry. However, when placing all but one detector at the so-called magic positions (MP; refs 23,24), only specific spatial frequency vectors of the object appear within a given correlation function of order $m$. In particular, if $m-1$ detectors are placed along the $x$-axis such that $\tilde{f}_{x} x_{j}=(j-2) /(m-1)$, $j=2, \ldots, m$, where $\tilde{f}_{x}=d_{x} /(\lambda L)$ is the spatial frequency associated with the lattice constant $d_{x}$, only those spatial frequency vectors $\mathbf{f}_{i}$ of the object appear in $g^{(m)}\left(\mathbf{r}_{1} ; M P_{x}\right)$ where the $x$-component fulfils the filtering condition $f_{i, x}=\kappa(m-1) \tilde{f}_{x}$, with $\kappa \in N_{0}$; an analogue filtering condition holds for the $y$-direction. As in 1D (ref. 24), the filtering process in $2 \mathrm{D}$ is a result of sum rules which hold for the $(m-1)$ th-roots of unity (see Supplementary Information).

As an example, we consider $g^{(4)}\left(\mathbf{r}_{1} ; M P_{x}\right)$ with three detectors located at the MP along the $x$-direction (see Fig. 1). In this case only the spatial frequency vectors $\zeta_{x}^{(4)}=\left\{\left(\begin{array}{l}3 \\ 1\end{array}\right),\left(\begin{array}{c}3 \\ -1\end{array}\right),\left(\begin{array}{l}0 \\ 2\end{array}\right)\right\}$ (in units of $\tilde{f}_{x}$ and $\left.\tilde{f}_{y}\right)$ appear in $g^{(4)}\left(\mathbf{r}_{1} ; M P_{x}\right)$, as only $f_{x}=1 \cdot 3 \tilde{f}_{x}$ (with $\kappa=1$ ) and $f_{x}=0 \cdot 3 \tilde{f}_{x}$ (with $\left.\kappa=0\right)$ match the filtering condition. Note that even though the fixed detectors are aligned at the MP along the $x$-direction, the corresponding components $f_{y}$ of the filtered $f_{x}$ also show up in $g^{(4)}\left(\mathbf{r}_{1} ; M P_{x}\right)$. 


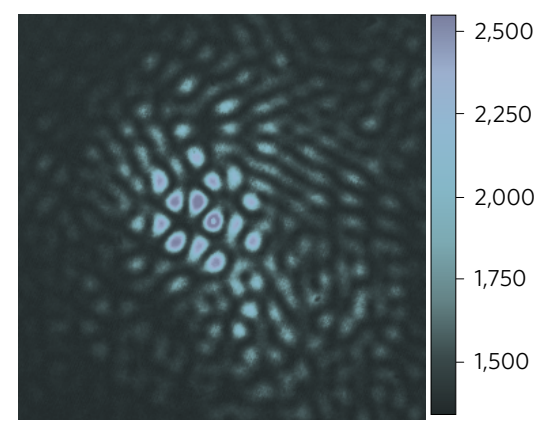

C

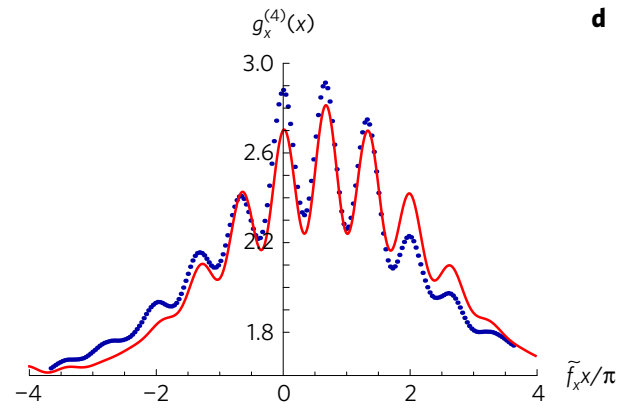

b
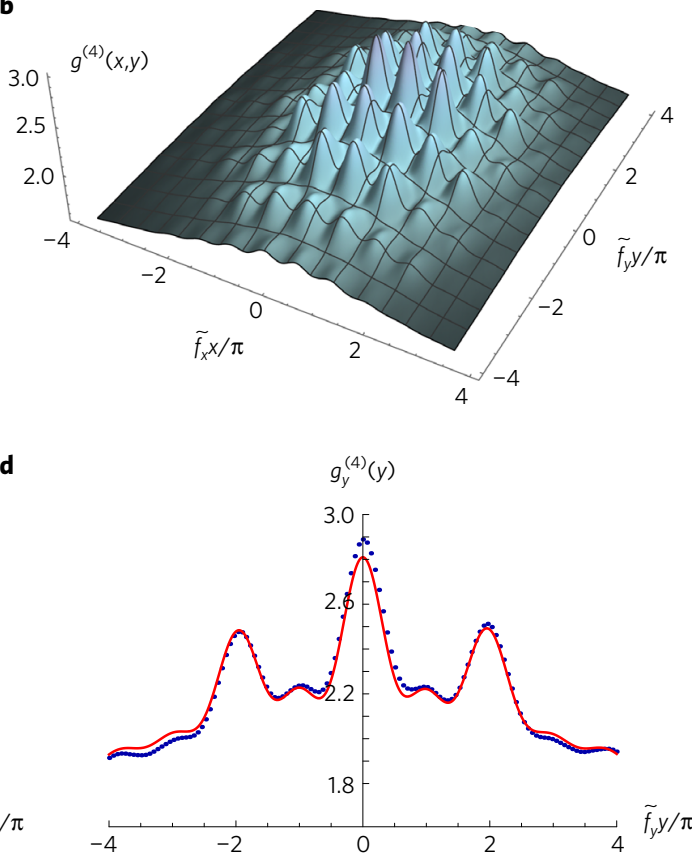

Figure 2 | Single-shot speckle pattern and experimentally derived fourth-order intensity correlation function $\boldsymbol{g}^{(4)}\left(r_{1} ; \boldsymbol{M} \boldsymbol{P}_{x}\right)$. a, Single-shot speckle pattern as measured by the CCD. $\mathbf{b}, 10,800$ single-shot speckle patterns are processed to obtain $g^{(4)}\left(\mathbf{r}_{1} ; M P_{x}\right)$ where three pixel detectors are located at the $M P$ along the $x$-direction. $\mathbf{c}, \mathbf{d}$, Cross-sections of $g^{(4)}\left(\mathbf{r}_{1} ; M P_{x}\right)$ (blue dotted curve) and of the best 2D fit to $g^{(4)}\left(\mathbf{r}_{1} ; M P_{x}\right)$ (red solid curve) in the $x$-direction (at $\tilde{f}_{y} y=0$ ) and in the $y$-direction (at $\tilde{f}_{x} x=(2 / 3) \pi$ ), respectively. The $2 \mathrm{D}$ fit allows one to access the set of spatial frequencies $\zeta_{x}^{(4)}$ displayed by $g^{(4)}\left(\mathbf{r}_{1} ; M P_{x}\right)$.

Table 1 | Theoretically expected and experimentally obtained spatial frequency vectors (in units of $\tilde{f}_{x}$ and $\tilde{f}_{y}$ ); colour labels correspond to the colours of the spatial frequency vectors shown in Fig. 1.

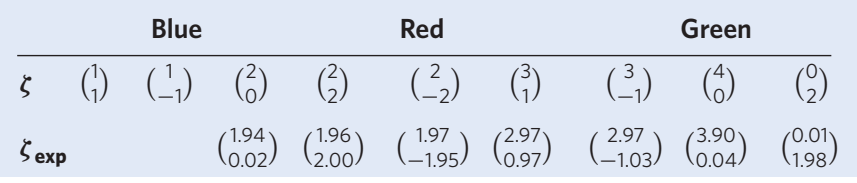

After evaluating $g^{(m)}\left(\mathbf{r}_{1} ; M P\right)$ for $m=3,4,5$, with the fixed detectors aligned at the MP along the $x$-axis as well as along the $y$-axis (resulting in a total of six 2D correlation functions), the set of spatial frequency vectors $\zeta_{\exp }$ of the benzene structure is derived from the best $2 \mathrm{D}$ fit to the experimental data (see Supplementary Information). As an example, the correlation function $g^{(4)}\left(\mathbf{r}_{1} ; M P_{x}\right)$, experimentally determined after evaluating 10.800 single-shot CCD images with three pixel detectors located at the MP along the $x$-direction, and corresponding fit functions are shown in Fig. $2 \mathrm{~b}-\mathrm{d}$.

The set of spatial frequency vectors $\zeta_{\exp }$ thus obtained is shown in Table 1. Note that since some spatial frequencies match the filtering condition for more than one correlation order they can be accessed from correlation functions $g^{(m)}\left(\mathbf{r}_{1} ; M P\right)$ of different orders $m$. However, it turns out that the values for $f_{x}$ and $f_{y}$ thus obtained deviate from each other by less than $1 \%$.

A systematical error for the experimentally derived set of spatial frequencies $\zeta_{\exp }$ originates from the finite size of the pixel detectors, as this prevents the $m-1$ fixed pixel detectors from being located exactly at the MP (assumed to be point-like). In our experiment, using a CCD camera with pixel size $13.5 \times 13.5 \mu \mathrm{m}^{2}$ at a distance of $L=275 \mathrm{~mm}$ behind the hole mask, this results in systematical errors for $f_{x}$ and $f_{y}$ of $3.6 \%$ and $6.3 \%$, respectively.

Note that according to the filtering condition the two spatial frequency vectors $\left(\begin{array}{l}1 \\ 1\end{array}\right)$ and $\left(\begin{array}{c}1 \\ -1\end{array}\right)$ (in units of $\tilde{f}_{x}$ and $\tilde{f}_{y}$ ) cannot be extracted from $g^{(m)}\left(\mathbf{r}_{1} ; M P\right)$, if $m \geq 3$. Hence, without measuring
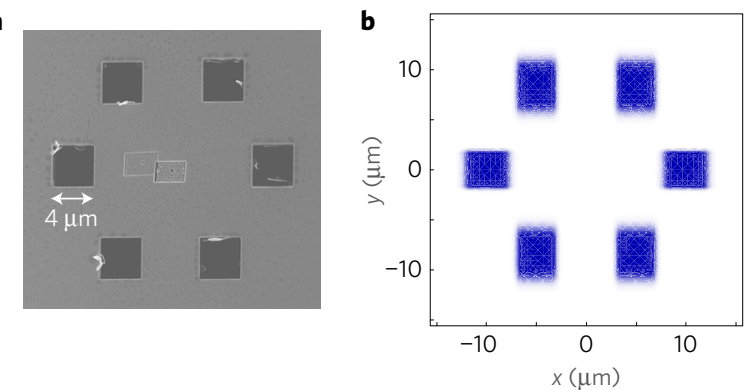

Figure $\mathbf{3}$ | Geometry of the artificial benzene molecule. a, Scanning electron microscopy image of the hole mask. $\mathbf{b}$, Image of the hole mask reconstructed after evaluating $g^{(m)}\left(\mathbf{r}_{1} ; M P\right)$ for $m=3,4$, 5, with the fixed detectors aligned at the MP along the $x$-axis as well as along the $y$-axis. The uncertainties of the source positions and sizes are displayed by a Gaussian-shaped colour gradient. The standard deviations are composed of the error for the source size (3.6\% and 6.3\% for $x$ - and $y$-direction, respectively) and the source positions (3.6\% and $6.3 \%$ for $x$ - and $y$-direction with respect to the centre of the structure).

$g^{(2)}\left(\mathbf{r}_{1} ; M P\right)$, four different possibilities to complete the set $\zeta$ from the set $\zeta_{\exp }$ are possible, containing either none, one of the two, or both spatial frequency vectors. However, for the investigated benzene structure only the set containing both spatial frequency vectors $\left(\begin{array}{l}1 \\ 1\end{array}\right)$ and $\left(\begin{array}{c}1 \\ -1\end{array}\right)$ provides a solution for the source arrangement in real space, resulting in a unique set $\zeta$ (see Supplementary Information).

Besides the positions of the sources, the correlation signals also contain information about the size of the sources as their finite extension translates into sinc-shaped envelopes in the $x$ and $y$-directions of $g^{(m)}\left(\mathbf{r}_{1} ; M P\right)$ (see Supplementary Information). Merging the extracted fit parameters - that is, the spatial frequencies of $g^{(m)}\left(\mathbf{r}_{1} ; M P\right)$ for $m=3,4,5$, as well as their envelopes-we are able to determine the entire benzene structure. The result is shown in Fig. 3. 
The reconstruction of the source geometry using our approach proves to be extremely robust against intensity and frequency fluctuations of the scattered $\operatorname{light}^{24}$. In particular, the method allows one to reconstruct the source arrangement in the complete absence of first-order coherence-for example, due to imperfect optics that leads to wave front distortions or vanishing coherent diffraction signals. Such aspects become increasingly important with decreasing wavelength. Thus imaging applications in the regime of hard X-rays, where first-order coherence is readily compromised by imperfect optical elements, will especially benefit from our imaging technique.

The method has further great potential for imaging with intense sources of X-rays, where a significant part of the emission and scattering occurs incoherently due to inelastic processes such as Compton scattering or fluorescence associated with the strong electromagnetic driving ${ }^{11,12}$. Therefore, the approach will become increasingly relevant for imaging with existing and future laser sources in the regime of hard X-rays, especially with respect to the ultimate goal of single-particle imaging (SPI) (refs 9,10). Powerful algorithms have been developed in the field of SPI (ref. 9) to deal with the single particles (or even molecules) being injected in random orientations into the beam, which could be transferred to our method when applied to SPI. Note that the method can also be combined with novel techniques such as macromolecular imaging from imperfect crystals $^{27}$, where the single-molecule scattering signal is discriminated with high signal-to-noise ratio against the coherent Bragg peaks from the crystalline structure. Moreover, applying the technique to the detection of resonance fluorescence as an intrinsically incoherent scattering process enables elementspecific imaging applications. Finally, it should be emphasized that the role of photons can be taken by any other bosonic particle. Using correlation functions based on anti-commutation relations, our imaging method could be further extended to fermions, such as pulsed beams of electrons, and possibly also pulsed neutrons from spallation neutron sources.

Data availability. The data that support the plots within this paper and other findings of this study are available from the corresponding author upon reasonable request.

\section{Received 24 March 2017; accepted 26 September 2017; published online 30 October 2017}

\section{References}

1. Chapman, H. N. et al. Femtosecond diffractive imaging with a soft-X-ray free-electron laser. Nat. Phys. 2, 839-843 (2006).

2. Chapman, H. N. et al. Femtosecond X-ray protein nanocrystallography. Nature 470, 73-77 (2011).

3. Seibert, M. et al. Single mimivirus particles intercepted and imaged with an X-ray laser. Nature 470, 78-81 (2011).

4. Loh, N. D. et al. Fractal morphology, imaging and mass spectrometry of single aerosol particles in flight. Nature 486, 513-517 (2012).

5. Kupitz, C. et al. Serial time-resolved crystallography of photosystem II using a femtosecond X-ray laser. Nature 513, 261-265 (2014).

6. Takahashi, Y. et al. Coherent diffraction imaging analysis of shape-controlled nanoparticles with focused hard X-ray free-electron laser pulses. Nano Lett. 13, 6028-6032 (2013).

7. Barke, I. et al. The 3D-architecture of individual free silver nanoparticles captured by X-ray scattering. Nat. Commun. 6, 6187 (2015).

8. Neutze, R., Wouts, R., van der Spoel, D., Weckert, E. \& Hajdu, H. Potential for biomolecular imaging with femtosecond X-ray pulses. Nature 406, 752-757 (2000).

9. Aquila, A. et al. The linac coherent light source single particle imaging road map. Struct. Dyn. 2, 041701 (2015).

10. Barty, A., Küpper, J. \& Chapman, H. N. Molecular imaging using X-ray free-electron lasers. Annu. Rev. Phys. Chem. 64, 415-435 (2013).

11. Slovik, J. M., Son, S.-K., Dixit, G., Jurek, Z. \& Santra, R. Incoherent X-ray scattering in single molecule imaging. New J. Phys. 16, 073042 (2014).
12. Gorobtsov, O. Y., Lorenz, U., Kabachnik, N. M. \& Vartanyants, I. A. Theoretical study of electronic damage in single-particle imaging experiments at X-ray free-electron lasers for pulse durations from 0.1 to 10 fs. Phys. Rev. E 91, 062712 (2015)

13. Hanbury Brown, R. \& Twiss, R. Q. A test of a new type of stellar interferometer on Sirius. Nature 178, 1046-1048 (1956).

14. Chapman, H. N. \& Nugent, K. A. Coherent lensless X-ray imaging. Nat. Photon. 4, 833-839 (2010).

15. Hanbury Brown, R. \& Twiss, R. Q. Correlation between photons in two coherent beams of light. Nature 177, 27-29 (1956).

16. Glauber, R. J. Nobel lecture: one hundred years of light quanta. Rev. Mod. Phys. 78, 1267-1278 (2006)

17. Glauber, R. J. The quantum theory of optical coherence. Phys. Rev. 130, 2529-2539 (1963)

18. Goodman, J. W. Statistical Optics (John Wiley \& Sons, 1985).

19. Baym, G. The physics of Hanbury Brown-Twiss intensity interferometry: from stars to nuclear collisions. Acta Phys. Pol. B 29, 1839-1884 (1998).

20. Singer, A. et al. Hanbury Brown-Twiss interferometry at a free-electron laser. Phys. Rev. Lett. 111, 034802 (2013).

21. Gorobtsov, O. Y. et al. Statistical properties of a free-electron laser revealed by Hanbury Brown-Twiss interferometry. Phys. Rev. A 95, 023843 (2017).

22. Thiel, C. et al. Quantum imaging with incoherent photons. Phys. Rev. Lett. 99, 133603 (2007)

23. Oppel, S., Büttner, T., Kok, P. \& von Zanthier, J. Superresolving multiphoton interferences with independent light sources. Phys. Rev. Lett. 109, 233603 (2012)

24. Classen, A. et al. Superresolving imaging of irregular arrays of thermal light sources using multiphoton interferences. Phys. Rev. Lett. 117, 253601 (2016).

25. Ackermann, W. et al. Operation of a free-electron laser from the extreme ultraviolet to the water window. Nat. Photon. 1, 336-342 (2007).

26. Goodman, J. W. Speckle Phenomena in Optics: Theory and Applications (Roberts and Company, 2007)

27. Ayyer, K. et al. Macromolecular diffractive imaging using imperfect crystals. Nature 530, 202-206 (2016).

\section{Acknowledgements}

R.S., T.M., A.C., D.B. and J.v.Z. gratefully acknowledge funding by the Erlangen Graduate School in Advanced Optical Technologies (SAOT) by the German Research Foundation (DFG) in the framework of the German excellence initiative. A.C. and D.B. gratefully acknowledge financial support by the Staedtler Foundation and the Cusanuswerk, Bischöfliche Studienförderung, respectively. We acknowledge support of the Helmholtz Association through project oriented funds. I.V. acknowledges the support of the Virtual Institute VH-VI-403 of the Helmholtz Association. Y.O. and S.W. acknowledge support by the Partnership for Innovation, Education and Research (PIER) between DESY and the University of Hamburg. We are grateful to the FLASH machine operators, to the technical staff at FLASH for excellent FEL conditions, and to Holger Meyer for his contributions to the design of the experimental set-up. We appreciate fruitful discussions with E. Weckert and H. N. Chapman.

\section{Author contributions}

J.v.Z., R.R. and I.A.V. conceived the experiment and coordinated the experimental efforts. R.S. and T.M. designed the experimental layout and provided post-measurement analysis and evaluation of experimental data. G.M. designed and built the experimental set-up. G.M., L.W., R.S., T.M., O.G., S.L., P.S. and I.Z. carried out the experiment at FLASH/DESY. A.C. and F.W. developed the idea of sequential spatial frequency filtering in $1 \mathrm{D}$ and $2 \mathrm{D}$ using higher-order intensity correlations. A.C. and D.B. developed quantum path analysis of the method and mathematical explanation for sub-Abbe resolution. A.B. installed the motor control of the diffusor stage. L.B. coordinated efforts to characterize diffusors and samples. B.F. provided silica particles for production of diffusors. K.S. prepared diffusors and thin film coatings. J.W. prepared the hole mask of the artificial benzene molecule used as sample. S.W. participated in preparation and characterization of diffusors. G.B. provided the operation of the beamline PG2 at FLASH. Y.O. participated in discussion of the theoretical basis of quantum imaging. W.W. provided the end station for measurements at the PG2 beamline. R.S., T.M., J.v.Z., R.R. and I.A.V. wrote the manuscript with contributions and improvements from all authors.

\section{Additional information}

Supplementary information is available in the online version of the paper. Reprints and permissions information is available online at www.nature.com/reprints. Publisher's note: Springer Nature remains neutral with regard to jurisdictional claims in published maps and institutional affiliations. Correspondence and requests for materials should be addressed to J.v.Z

\section{Competing financial interests}

The authors declare no competing financial interests. 